Original Article

\title{
FORMULATION, STABILITY TEST AND IN VITRO PENETRATION STUDY OF TRANSETHOSOMAL GEL CONTAINING GREEN TEA (CAMELLIA SINENSIS L. KUNTZE) LEAVES EXTRACT
}

\section{DELLY RAMADON*, SESHIANA SEBTI PRAMESTI, EFFIONORA ANWAR}

Laboratory of Pharmaceutics and Pharmaceutical Technology, Faculty of Pharmacy, Universitas Indonesia, Depok, West Java, Indonesia Email: delly.ramadon@farmasi.ui.ac.id

Received: 18 May 2017, Revised and Accepted: 22 Aug 2017

\section{ABSTRACT}

Objective: The aim of this study was to increase penetration of epigallocatechin gallate (EGCG) from the extract using transethosomal gel.

Methods: Transethosomes (TE) formulae were made using thin layer hydration method with different concentration of green tea extract which was equivalent to 1\% (F1), 1.5\% (F2), and 2\% (F3) of EGCG. F1 was the chosen formula to be incorporated into a gel as a transethosomal gel (TEG). A gel containing green tea extract was also made as a control called as non-transethosomal gel (NTEG). A stability test and in vitro penetration study of gels using Franz diffusion cell were performed.

Results: F1 was the chosen formula because it had a spherical shape, a particle size of $112.14 \pm 2.19 \mathrm{~nm}$, PDI of $0.166 \pm 0.03$, a zeta potential of$52.05 \pm 1.34 \mathrm{mV}$, and \%EE of 58.06 $\pm 0.08 \%$. Stability test results showed that TEG more stable than NTEG. The amount of EGCG penetrated from TEG and NTEG were $1391.16 \pm 34.89 \mu \mathrm{g} / \mathrm{cm}^{2}$ and $485.29 \pm 14.49 \mu \mathrm{g} / \mathrm{cm}^{2}$, respectively $(p<0.05)$. The lag time for TEG was around $0.99 \pm 0.2 \mathrm{~h}$, while NTEG was $8.69 \pm 0.2 \mathrm{~h}(p<0.05)$.

Conclusion: It can be concluded that transethosomes can improve gel stability and increase the amount of EGCG penetrated through the skin.

Keywords: EGCG, Green tea leaves extract, In vitro penetration, Transethosomes, Transethosomal gel

(C) 2017 The Authors. Published by Innovare Academic Sciences Pvt Ltd. This is an open access article under the CC BY license (http://creativecommons.org/licenses/by/4.0/) DOI: http://dx.doi.org/10.22159/ijap.2017v9i5.20073

\section{INTRODUCTION}

Nowadays, nanovesicles usage in pharmaceutical technology has been developed. It can be applied in many kinds of drug delivery system as in transdermal drug delivery system (TDDS). TDDS uses the skin as an alternative way to deliver drugs into the systemic circulation. Some advantages of drug delivery via the transdermal route are avoiding digestive degradation, passing the hepatic metabolism, and it can be used to deliver many kinds of medicinal products like chemical substances or natural products [1].

One of the nanovesicles that can be formulated into a transdermal dosage form is transethosomes (TE) [2]. TE is an evolution of deformable vesicles that was developed by Song et al. in 2012 [2]. TE has more advantages than transfersomes and ethosomes, such as higher elasticity and flexibility than both the previous vesicles [3]. $\mathrm{TE}$ is composed of phospholipids, ethanol, edge activators (surfactants) and penetration enhancers. High elasticity of TE is influenced by a combination of ethanol and edge activator $[3,4]$. Also, it has been used for chemical substances or natural products.

Currently, the utility of natural products for medical purposes is also increasingly in demand. One of the abundant natural materials and has many health benefits is green tea leaves (Camellia sinensis L. Kuntze). Green tea contains approximately 4000 bioactive components, and the most dominant are polyphenols [5]. Polyphenols in green tea are mostly from catechin groups, and they are about $30 \%$ of the total content of green tea bioactive components $[6,7]$. Examples of catechin derivative compounds in green tea are (-)-epigallocatechin gallate (EGCG) (8-12\%), (-)epigallocatechin (EGC) (3-6\%), (-)-epicatechin 3-gallate (ECG) (3$6 \%$ ), and epicatechin (EC) (1-3\%) [8]. these compounds have a high antioxidant activity that is beneficial in preventing many diseases associated with increasing of oxidative stress such as cancer, diabetes, and cardiovascular disease [9-11]. Based on some of these properties, green tea extract is suitable to be formulated into a transdermal dosage form to give the beneficial effects to the body.

Bioactive components in green tea leaves should be able to penetrate through the skin to provide an optimal antioxidant effect.
However, green tea catechin such EGCG has a problem to penetrate through the skin. It has a high polarity $(\log P=0.48)[12]$ and a large molecular mass $(458.37 \mathrm{Da})$ that can inhibit its penetration into the skin [13]. One of strategy to overcome these problems is formulating the green tea extract into a lipid vesicle such as TE [2].

Green tea extract or EGCG had been formulated into some types of vesicles, such as liposomes [14], transfersomes [15], and nanoparticles [16, 17]. However, there was not research on formulating the green tea into TE. The formulation of green tea extract in TE is expected to be an alternative way to increase penetration of EGCG from the extract. In this study, green tea leaves extract (Camellia sinensis L. Kuntze) was formulated into TE, and the marker analyzed was EGCG. Then a chosen TE formula would be incorporated into a gel dosage form. The stability test was conducted to the gels prepared. Additionally, an in vitro penetration test using Franz diffusion cell was performed to evaluate the penetration of EGCG through the skin. EGCG was analyzed by thin layer chromatography (TLC) densitometer.

\section{MATERIALS AND METHODS}

\section{Materials}

Green tea leaves extract containing $42.80 \%$ EGCG was purchased from Andy Biotech (Xi'an, China). EGCG secondary standard (purity 92.4\%) was supplied by Sigma-Aldrich, (Singapore, Singapore). Lipoid ${ }^{\circledR}$ P30 was kindly gifted by Lipoid $\mathrm{GmbH}$ (Ludwigshafen, Germany). Span 80 was obtained from Croda (Nexus, Singapore). Carbopol $^{\circledR} 940$ was offered by Lubrizol (Wanchai, Hongkong). Ethanol was purchased from Merck (Darmstadt, Germany) and propylene glycol from Dow Chemical Co. (Michigan, America). Female Sprague-Dawley rats were provided by Bogor Agricultural University (Bogor, Indonesia). Other solvents and reagents were the analytical grade or better.

\section{Preparation of transethosomes}

TE was formulated into three formulae with different concentration of green tea leaves extract as seen in table 1 . TE was made by thin layer hydration method referred to Ma et al. [4] with slight 
modification. Lipoid P30 and Span 80 were dissolved in dichloromethane. The organic solvent was then evaporated by using a rotary evaporator at a speed of $150 \mathrm{rpm}$ under a vacuum condition at a temperature of $40{ }^{\circ} \mathrm{C}$ until a thin layer was formed. After $24 \mathrm{~h}$, the thin layer was then hydrated with a mixture solvent of ethanol- phosphate buffer solution pH 5.5 (2:8), which previously have been mixed with green tea leaves extract. The hydration process was done in a rotary evaporator for one $h$ at a speed of $100 \mathrm{rpm}$ at $37^{\circ} \mathrm{C}$ without vacuum condition. The TE suspension was then sonicated for $20 \mathrm{~min}$ and stored at a temperature of $5^{\circ} \mathrm{C}$ before used.

Table 1: Formulation of transethosomes containing green tea leaves extract

\begin{tabular}{lll}
\hline Ingredients & F1 & F2 \\
\hline Green tea leaves extract (g) & $2.3^{(\mathrm{a})}$ & $3.5^{(\mathrm{b})}$ \\
Lipoid P30 (g) & 4.0 & 4.0 \\
Span 80 (g) & 0.7 & 4.0 \\
Ethanol-phosphate buffer solution pH 5.5 (2:8) $(\mathrm{ml})$ & Ad 100 & 0.7 \\
\hline
\end{tabular}

Note: Extracts are equal to EGCG: (a) 1\%, (b) 1.5\%, and (c) $2 \%$

\section{Morphology of transethosomes}

Morphology of TE was viewed by transmission electron microscope (TEM) (JEOL JEM 1400). A sample $(1.0 \mathrm{ml})$ was dispersed in demineralized water, and then the solution was dropped onto the carbon-coated copper grid. After that, 1\% phosphotungstic acid solution was dropped into the sample [2].

\section{Entrapment efficiency}

Entrapment efficiency (\%EE) of TE was conducted by a direct method. As a marker of the extract, EGCG concentration was measured to obtain the \%EE. TE suspension was put in a centrifuge tube and centrifuged at $13000 \mathrm{rpm}$ for $2 \times 30 \mathrm{~min}$. There were supernatant and precipitated TE. Then, the precipitated TE was collected, added with chloroform and revortexed to disrupt the vesicle. After that, ethanol was then added to the mixture to dissolve the EGCG. The solution obtained was spotted on silica gel plate to analyze the concentration of EGCG. The concentration of the drug entrapped in TE was calculated by the following formula [18]:

$$
\text { Entrapment efficiency }(\%)=\frac{\mathrm{Qe}}{\mathrm{Qt}} \times 100 \%
$$

$\mathrm{Qe}=$ concentration of EGCG entrapped in sediment $(\mu \mathrm{g} / \mathrm{ml})$

$\mathrm{Qt}=$ total concentration of EGCG added in suspension $(\mu \mathrm{g} / \mathrm{ml})$

\section{Particle size distribution, polydispersity index, and zeta potential}

Particle size, polydispersity index, and zeta potential were measured by using a dynamic light scattering method of particle size analyzer
(Malvern Zetasizer). Each sample was diluted by demineralized water then analyzed at a temperature of $25^{\circ} \mathrm{C}$ [2].

\section{Deformability index}

Deformability index was determined by the extrusion method. TE samples were injected through a cellulose membrane with $100 \mathrm{~nm}$ pores at $0.5 \mathrm{MPa}$. Deformability of TE was calculated using the formula below [2]:

$$
E=j\left(\frac{r v}{r p}\right)^{2}
$$

$\mathrm{E}=$ deformability index

$\mathrm{J}=$ volume of TE that can flow through the membrane

$r_{v}=$ size of vesicles after extrusion

$r_{p}=$ average size of membrane pores

\section{Gel formulation}

There were two gels prepared, i.e. transethosomal gel (TEG) and non-transethosomal gel (NTEG) as seen in table 2. Carbopol was dispersed in demineralized water, and then triethanolamine was added to form a viscous gel base. Next, the mixture was homogenized using a homogenizer at a speed of $1000 \mathrm{rpm}$. During stirring, propylene glycol and TE suspension were added into the TEG formula. To make NTEG, green tea leaves extract which was previously dissolved in a mixture of ethanol-phosphate buffer solution $\mathrm{pH}$ 5.5, was mixed into the separate gel base, and then it was homogenized with a homogenizer at a speed of $1000 \mathrm{rpm}$ for 10 min.

Table 2: Gel formulation

\begin{tabular}{lll}
\hline Ingredients & Concentration (\% w/w) \\
\cline { 2 - 3 } & TEG & NTEG \\
\hline Carbopol 940 & 1.0 & 1.0 \\
Triethanolamine & 0.6 & 0.6 \\
Propylene glycol & 12.5 & 12.5 \\
Transethosomes suspension equal to EGCG & 0.5 & - \\
Green tea leaves extract equal to EGCG & - & 0.5 \\
Demineralized water & ad 100 & ad 100 \\
\hline
\end{tabular}

Note: (TEG): Transethosomal Gel, (NTEG): Non-transethosomal Gel

\section{Gel evaluation and stability test}

After finishing the preparation, gels were immediately evaluated their homogeneity, organoleptic, $\mathrm{pH}$, and rheological property. Then, the gels were stored at three different temperatures, i.e. low temperature $\left(4 \pm 2{ }^{\circ} \mathrm{C}\right)$, room temperature $\left(27 \pm 2{ }^{\circ} \mathrm{C}\right)$, and hot temperature $\left(40 \pm 2{ }^{\circ} \mathrm{C}\right)$ for $12 \mathrm{w}$. The organoleptic and $\mathrm{pH}$ of the gels were checked every two w, while the rheological property was investigated at week 0 and 12 . A cycling test was also performed by storing the gels at a temperature of $4{ }^{\circ} \mathrm{C}$ for $24 \mathrm{~h}$ and then transferred to a storage temperature of $40{ }^{\circ} \mathrm{C}$ for $24 \mathrm{~h}$, called as one cycle. The cycling test was performed with repetition as much as six cycles [19].

\section{In vitro penetration test}

In this research, an in vitro penetration test was conducted by using a Franz diffusion cell. The membrane used was the abdomen skin of female Sprague-Dawley rats (2-3 mo), weighing $\pm 150-200$ g. Rats were acclimated in an animal house for two weeks before the study. Rats were housed four rats per cage at a room temperature of $27 \pm 2{ }^{\circ} \mathrm{C}$ with 
an alternating $12 \mathrm{~h}$ light and dark cycle and given free access to food and water. All methods for euthanizing the animals were approved by Ethical Clearance Committee of Cipto Mangunkusumo Public Hospital, Faculty of Medicine-Universitas Indonesia No. 418/UN.2F1/ETIK/2016. The hair of rat skin was removed before used in the test. The receptor compartment was filled with $15 \mathrm{ml}$ of phosphate buffer solution $\mathrm{pH} 5.5$, then stirred with a magnetic stirrer at a speed of $250 \mathrm{rpm}$. Receptor compartment temperature was maintained at $37 \pm 1$ ${ }^{\circ} \mathrm{C}$. Then, a rat skin with a diffusion area of $2.01 \mathrm{~cm}^{2}$ was placed between the donor and the receptor compartment with dermal side facing the receptor compartment. A sample gel $(1 \mathrm{~g})$ containing green tea extract that was equivalent to 1\% EGCG was then applied onto the rat skin surface. The test was run for $24 \mathrm{~h}$ and samples were taken from the receptor compartment as much as $3.0 \mathrm{ml}$ at several time intervals $(1,3$, $5,7,8,10,12,14,16,18,20,22$, and $24 \mathrm{~h}$ ). At each sampling, $3.0 \mathrm{ml}$ of fresh phosphate buffer solution $\mathrm{pH} 5.5$ was added to the receptor solution to maintain sink condition. Then the EGCG concentration was measured by a TLC method. The cumulative amount and flux of EGCG penetrated were then calculated [20].

\section{Thin layer chromatography condition}

All samples were spotted on a silica gel $60 \mathrm{~F}_{256}$ plates and eluted using dichloromethane-acetone-formic acid (8:7:1) as the eluent. The plates were then analyzed by TLC scanner III (CAMAG) at a wavelength of $275 \mathrm{~nm}$.

\section{Statistical analysis}

Statistical analysis of data was analyzed by GraphPad Prism 6.0 via unpaired t-tests with significance level was set at $P<0.05$.

\section{RESULTS AND DISCUSSION}

One of strategy to increase drug penetration is entrapping drugs into nanovesicles [21]. Since conventional liposomes has some weaknesses, there were many developments of vesicles to obtain a better delivery. TE as a novel vesicle, an advanced form of transfersomes and ethosomes, is expected to be the best way to overcome drug penetration problems. To penetrate through the deeper layers of the skin, TE must have some characteristics. For instance, it should be small, elastic, and it must have a high drug entrapment.

\section{Preparation of transethosomes}

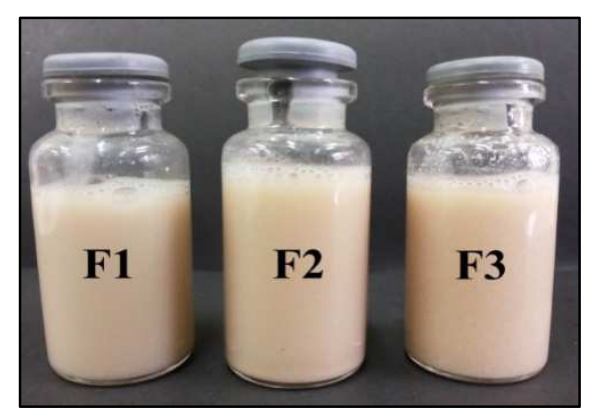

Fig. 1: The appearance of transethosomes formulae containing green tea extract

In this study, preparation of TE was done by thin film hydration method because it provides a higher drug loading for multilamellar vesicles (MLV) than other methods [22]. This method has been optimized and observed by Zaafarany et al. [23]. The preparation of a thin film in the rotary evaporation method offers a large surface area that can give a perfect hydration of vesicles [23]. All TE formulae prepared can be seen in fig. 1 .

\section{Effect of edge activator to vesicles morphology}

The morphological observation showed that all formulae had an irregular spherical shape as seen in fig. 2. This form caused by a combination of ethanol and Span 80 which can lead to a rearrangement of lipid bilayer vesicles composition [3]. It shows the lamellar and core of each vesicle under the microscope. The dark color in the middle of vesicle showed that the extract was entrapped in the core of TE because it is a hydrophilic substance. When a drug is dissolved in the mixture of ethanol and phosphate buffer solution pH 5.5, it has a high polarity. Then, it produces electrons that can be captured by TEM. So, the properties of substances will determine their entrapment position in TE.

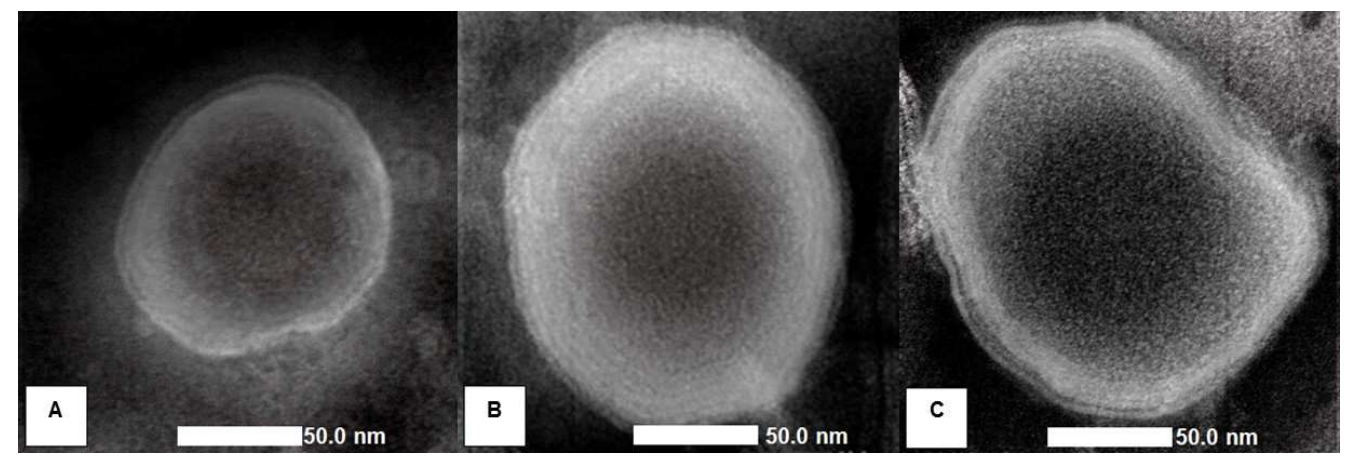

Fig. 2: The morphology of transethosome viewed using TEM with 150000 magnification. (A) F1, (B) F2 and (C) F3

\section{Effect of drug concentration to entrapment efficiency}

Besides morphology of vesicles, \%EE is an important parameter for vesicles. TE must have a high entrapment of EGCG to be incorporated into a gel dosage form. The higher EGCG entrapped in the TE, the less amount of TE needed in gel formulation. In this study, the results revealed that $\mathrm{F} 1$ had the highest \%EE among other formulae. However, based on the results, it is known that the higher concentration of extract in TE formula, the lower \%EE obtained. This result is similar to Zaafarany et al. [23], they demonstrated that a further increase in drug concentration led to a significant decrease in \%EE. This phenomenon caused by the saturation of lipid components and hydrophilic part of the TE by the extract used because the vesicles of TE have a limitation capacity of drug entrapment [24].
Effect of drug concentration and polydispersity index to particle size

Another important characteristic for enhancing drug penetration is particle size of vesicles. The results of particle size, polydispersity index and zeta potential measurements of all TE formulae can be seen in the summary of TE characterization results as seen in table 3. Based on results above, all TE formulae were in nano-size. There was a correlation between the increase in drug concentration and particle size. The rise of extract concentration could cause higher collisions between particles. It is directly proportional to the increase of particle size of TE. Collisions between particles may lead to the aggregation process of the particles which allow increasing the particle size [25]. It results in the heterogeneity of polydispersity index (PDI) value. The higher concentration of extract formulated, the bigger PDI of a formula. PDI is a 
level of heterogeneity of particle size in a formula. When a formula has a small value of PDI, it indicates that the formula is a homogenous vesicle. Finally, this leads to the decrease of particle size.

\section{Zeta potential}

To keep the particle in a stable condition or avoid the aggregation, TE must have a good zeta potential which is more positive than $+30 \mathrm{mV}$ or more negative than-30 mV. Zeta potential is an electrostatic charge which provides repulsion between the particles [25]. Zeta potential is increasingly positive or negative that indicates the magnitude of the repulsive force between charged particles [26]. In this study, green tea extract was a drug model, and EGCG is the marker of the extract. EGCG is a negatively charged substance, and this is accordance with the charge of TE in this study. Sou [27] found that vesicles with zeta potential that is less than- $30 \mathrm{mV}$ tend to have high entrapment capacity.

\section{Deformability index}

A vesicle should be elastic to penetrate the deeper layers of the skin [2]. The deformability index measurement of F1, F2, and F3 were $1.11 \pm 0,00,1.46 \pm 0,00$, and $2.14 \pm 0,00$, respectively. A surfactant as an edge activator gives a deformable characteristic to vesicles [23]. This property can make the TE changes their shape easily into a very tiny space between cells of stratum corneum. Deformability of TE is influenced by the presence of Span 80 as an edge activator and ethanol as penetration enhancers. As an edge activator, Span 80 can destabilize lipid bilayer thereby decreases the rigidity of the lipid bilayer [28]. Ethanol can interact with the polar part of the phospholipid that causes a reduction in the melting point of phospholipids so that it can improve the fluidity and permeability of lipid bilayer membrane and permeability of membranes [29].

Table 3: Summary results of transethosome characterization

\begin{tabular}{|c|c|c|c|c|c|}
\hline Formula & Morphology & Dmean volume (nm) & Polydispersity index & Zeta potential (mV) & Entrapment efficiency (\%) \\
\hline F1 & Spheric & $112.14 \pm 2.19$ & $0.163 \pm 0.01$ & $-52.05 \pm 1.34$ & $58.06 \pm 0.09$ \\
\hline F2 & Spheric & $132.94 \pm 5.42$ & $0.175 \pm 0.02$ & $-63.6 \pm 6.93$ & $41.15 \pm 0.13$ \\
\hline F3 & Spheric & $179.49 \pm 1.76$ & $0.272 \pm 0.01$ & $-62.6 \pm 5.66$ & $33.42 \pm 0.22$ \\
\hline
\end{tabular}

Note: All values were represented as mean $\pm \operatorname{SD}(n=3)$

\section{Gel formulation}

Based on table 3, F1 was chosen formula to be incorporated into a gel dosage form because it had an almost spherical shape, smallest $D_{\text {mean }}$ volume, lowest polydispersity index $(<0.5)$, zeta potential $> \pm 30$ $\mathrm{mV}$, and the highest \%EE.

\section{Gel evaluation and stability test}

At week 0, all gels prepared showed a homogeneity in appearance. TEG showed a whitish brown color, while the NTEG was transparent brown as seen in fig. 3. pH of TEG and NTEG were 5.76 and 5.72, respectively. And the rheological property of all gels was plastic thixotropic. After 12 $\mathrm{w}$ storage, all gels were still homogeneous, and the $\mathrm{pH}$ of all gels was around 5.40-5.80. The results were still in the range of skin pH (4.5-6.5). There was not a change in the rheological properties too. Also, the color of TEG in all temperature storage did not change.

However, there was a color change in NTEG at the room and hot temperature storage. At the room temperature, NTEG showed a darker color in the sixth w. And, the color change at the hot temperature storage was in the fourth $\mathrm{w}$. The results revealed that TE could protect the green tea extract or EGCG from the oxidation. Verma et al. [30] revealed that formulating drugs into nanovesicles is one of strategy to enhance their stability. The vesicle keeps the drugs in the core of vesicle, so it cannot be exposed to the environment condition.

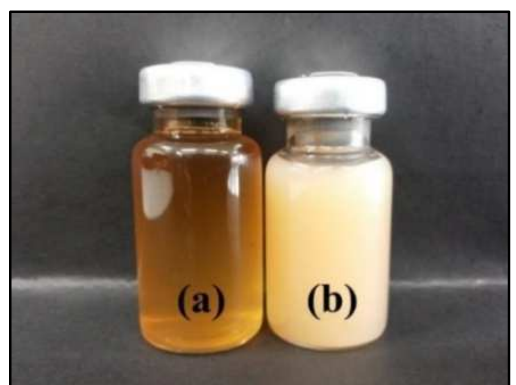

Fig. 3: Physical appearance of (a) NTEG and (b) TEG

\section{In vitro penetration test}

In vitro skin penetration test has been commonly used to measure the rate of drug release to reflect the combination effect of several physical and chemical parameters, including solubility and particle size of the active ingredients [21]. Based on the results as seen in fig. 4 , the cumulative amount of EGCG penetrated from TEG and NTEG were $1391.17 \pm 34.89 \mu \mathrm{g} / \mathrm{cm}^{2}$ and $485.29 \pm 14.49 \mu \mathrm{g} / \mathrm{cm}^{2}$, respectively $(p<0.05)$. Flux values of TEG and NTEG were $61.47 \pm 1.66 \mu \mathrm{g} / \mathrm{cm}^{2} . \mathrm{h}$ and $31.69 \pm 1.02 \mu \mathrm{g} / \mathrm{cm}^{2}$. h, respectively $(p>0.05)$ as seen fig. 5. Flux can be obtained from the slope in fig. 4 , taken in the steady state condition that follows the rule of Fick's law [25]. There was an effect of TE to the time penetration of EGCG through the skin. The lag time for TEG was around $0.99 \pm 0.2 \mathrm{~h}$, while NTEG was $8.69 \pm 0.2 \mathrm{~h}$ $(p<0.05)$. The results showed that TE could increase the amount of EGCG penetrated and reduce the lag time significantly. However, the increase of flux of TEG was not significant when compared to NTEG.

In vitro penetration study revealed that TEG exhibited superiority on the amount of EGCG penetrated compared to NTEG. It has been known that EGCG is a hydrophilic compound with a high polarity and molecular size. EGCG in TEG can more easily penetrate through the skin because it was entrapped inside the TE vesicle. TE can penetrate through the skin very well because it is composed of phospholipids which are similar to the composition of the lipid bilayer membrane in the stratum corneum. The results explained that there was an impact of partition coefficient in drug penetration due to the entrapment of EGCG from green tea in TE. The partition coefficient is one of the factors that can affect drug penetration [31-33]. Phospholipid which has the same partition coefficient with the lipid bilayer in the stratum corneum, helps the EGCG entrapped to penetrate easily through the skin. When the EGCG was not entrapped inside the vesicles, it would be blocked by the hydrophobic lipid bilayer membrane because it is very hydrophilic compounds.

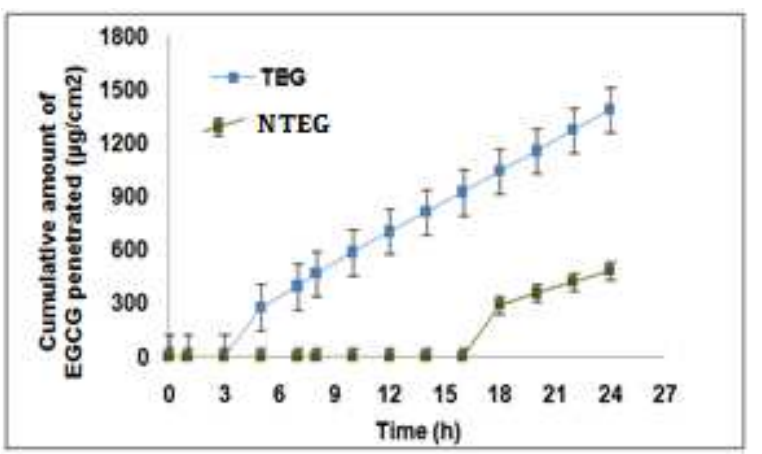

Fig. 4: Cumulative amount of EGCG penetrated from TEG and NTEG (control). (mean \pm SD $\left.(n=3) ;{ }^{*} P<0.05\right)$ 


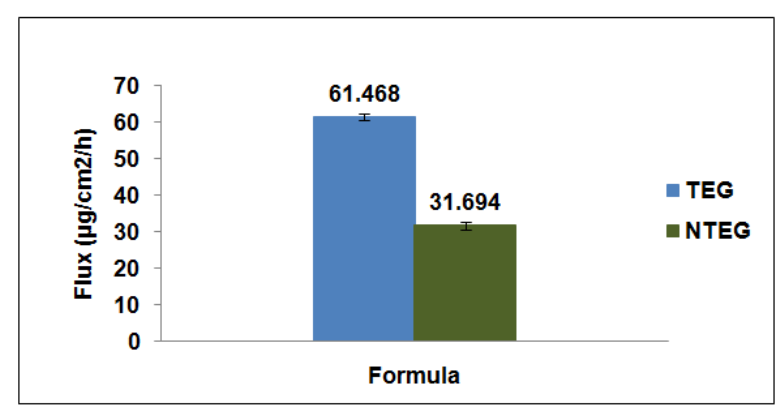

Fig. 5: Flux of TEG and NTEG (control). mean $\pm S D(n=3)$

Furthermore, as explained above, the presence of Span 80 as an edge activator could also increase the flexibility of TE. Span 80 as an edge activator can destabilize the vesicle membrane, so it can more easily deform through the small gap [8]. Additionally, a high concentration of ethanol in TE can be a penetration enhancer, and it facilitates EGCG to penetrate through the skin. The ethanol can also work to increase the penetration of the vesicles through the skin by reducing the rigidity of the membrane so that it can increase skin permeability [23]. Thus, the compact structure of skin, which is represented by three distinct layers (stratum corneum, the viable dermis, and the papillary layers of the dermis), is not a problem anymore for many drugs [34].

\section{CONCLUSION}

This research showed that many factors can influence drug entrapment efficiency of TE formula. It was also known that transethosomes caould enhance the stability of the extract in a gel dosage form. Additionally, it could improve the amount of EGCG penetrated and lag time of penetration but not for the flux. It can be summarized that transethosomes can be considered as an alternative solution to increase gel stability and penetration through the skin.

\section{ACKNOWLEDGEMENT}

The authors are highly thankful to Universitas Indonesia who had given financial support for this research, particularly for PITTA Research Grants 2016.

\section{CONFLICT OF INTERESTS}

The authors have declared no conflict of interest

\section{REFERENCES}

1. Lakshmi D, Prasanthi D. Vesicles-mechanism of transdermal permeation: a review. Asian J Pharm Clin Res 2012;5:18-25.

2. Song CK, Balakrishnan P, Shim C, Chung S, Chong S, Kim D. A novel vesicular carrier, transethosome, for enhanced skin delivery of voriconazole: characterization and in vitro/in vivo evaluation. Colloids Surf B 2012;92:299-304.

3. Ascenso A, Raposo S, Batista C, Cardoso P, Mendes T, Praça FG, et al. Development, characterization, and skin delivery studies of related ultradeformable vesicles: transfersomes, ethosomes, and transethosomes. Int J Nanomed 2015;10:5837-51.

4. Ma M, Wang J, Guo F, Lei M, Tan F, Li N. Development of nanovesicular systems for dermal imiquimod delivery: physicochemical characterization and in vitro/in vivo evaluation. J Mater Sci Mater Med: Materials Med 2015;26:1-11.

5. Anand J, Upadhyaya B. Biochemical characterization and pharmacognostic evaluation of purified catechins in green tea (Camellia sinensis) cultivars of India. 3 Biotechnology 2015;5:285-94.

6. Hassan SMA, Hussein AJ, Saeed AK. Role of green tea in reducing epidermal thickness upon ultraviolet light-b injury in BALB/c mice. Hindawi Adv Biol 2015;1-6. http://dx.doi.org/10.1155/2015/890632

7. Reddyvari H, Govatati S, Matha SK, Korla SV, Malempati S, Pasupuleti SR, et al. Therapeutic effect of green tea extract on alcohol. J Adv Res 2017;8:289-95.
8. Primavesi L, Piantanida M, Pravettoni V. Studying tea polyphenols and their protective effects on skin. Polyphenols Hum Health Dis 2013;1:849-59.

9. Bansal S, Syan N, Mathur P, Choudhary S. Pharmacological profile of green tea and its polyphenols: a review. Med Chem Res 2012;21:3347-60.

10. Mereles D, Hunstein W. Epigallocatechin-3-gallate (EGCG) for clinical trials: more pitfalls than promises? Int J Mol Sci 2011;12:5592-603.

11. Singh BN, Shankar S, Srivastava RK. Green tea catechin, epigallocatechin-3-gallate (EGCG): Mechanisms, perspectives and clinical applications. Biochem Pharmacol 2011;82:1807-21.

12. Patel AR, Velikov KP. Non-covalent interactions of green tea polyphenols: Source of novel colloidal structures. Nova Biomed; 2013. p. 151-66.

13. Belo SE, Gaspar LR, Maia PMBG, Marty J. Skin penetration of epigallocatechin-3-gallate and quercetin from green tea and ginkgo biloba extracts vehiculated in cosmetic formulations. Skin Pharmacol Physiol 2009;22:299-304.

14. Fang JY, Lee WR, Shen SC, Huang YL. Effect of liposome encapsulation of tea catechins. J Derm Sci 2006;42:101-9.

15. Avadhani KS, Manikkath J, Tiwari $M$, Chandrasekhar $M$, Godavarthi A, Vidya SM, et al. Skin delivery of epigallocatechin3-gallate (EGCG). Drug Delivery 2017;24:61-74.

16. Manea AM, Andronescu C, Meghea A. Green tea loaded into solid lipid nanoparticles. UPB Sci Bull 2014;76:125-36.

17. Yi S, Wang Y, Huang Y, Xia L, Sun L, Lenaghan SC, et al. Tea nanoparticles for immunostimulation. J Biomed Nanotechnol 2014;10:1016-29.

18. Chen W, Hu S. Suitable carriers for encapsulation and distribution of endostar: comparison of endostar-loaded particulate carriers. Int J Nanomed 2011;6:1535-41.

19. Bajaj S, Singla D, Sakhuja N. Stability testing of pharmaceutical products. J Appl Pharm Sci 2012;2:129-38.

20. Batchelder RJ, Calder RJ, Thomas CP, Heard CM. In vitro transdermal delivery of the major cathecins and caffeine from extract of Camellia sinensis. Int J Pharm 2004;283:45-51.

21. Barry BW. Novel mechanisms and devices to enable successful transdermal drug delivery. Eur J Pharm Sci 2001;14:101-14.

22. Morsi NM, Aboelwafa AA, Dawoud MHS. Improved bioavailability of timolol maleate via transdermal transfersomal gel: statistical optimization, characterization, and pharmacokinetic assessment. J Adv Res 2016;7:691-701.

23. El Zaafarany GM, Awad GAS, Holayel SM, Mortada ND. Role of edge activators and surface charge in developing ultradeformable vesicles with enhanced skin delivery. Int J Pharm 2010;397:164-72.

24. Ning MY, Guo YZ, Pan HZ, Yu HM, Gu ZW. Preparation and evaluation of proliposomes containing clotrimazole. Chem Pharm Bull 2005;53:620-4.

25. Sinko PJ. Martin's physical pharmacy and pharmaceutical sciences. 5th Ed. USA: Lippincott Williams and Wilkins; 2006.

26. Malvern Instruments. Dynamic Light Scattering Common Terms Defined; 2011. p. 1-6.

27. Sou K. Electrostatics of carboxylated anionic vesicles for improving entrapment capacity. Chem Phys Lipids 2011;164:211-5.

28. Elsayed MMA, Cevc G. Biochimica et biophysica acta the vesicleto-micelle transformation of phospholipid-cholate mixed aggregates: A state of the art analysis including membrane curvature effects. BBA-Biomembranes 2011;1808:140-53.

29. Nandure HP, Puranik P, Giram P, Lone V. Ethosome: a novel drug carrier. Int J Pharm Res Allied Sci 2013;2:18-30.

30. Verma P, Pathak K. Nanosized ethanolic vesicles loaded with econazole nitrate for the treatment of deep fungal infections through topical gel formulation. Nanomedicine: NBM 2012;8:489-96.

31. Anwar E, Ramadon D, Harmita H. Formulation and evaluation of gel and emulgel of chili extract (Capsicum frutescens L.) as topical dosage forms. Int J Pharm Pharm Sci 2014;6(Suppl 3):13-6.

32. Ramadon D, Aisha GW, Anwar E. Novel transdermal ethosomal gel containing green tea (Camellia sinensis L. Kuntze) leaves extract: formulation and in vitro penetration study. J Y Pharm 2017;9:1-5. 
33. Anwar E, Utami TD, Ramadon D. Transfersomal gel containing green tea (Camellia sinensis L. Kuntze) leaves extract: increasing in vitro penetration. Asian J Pharm Clin Res 2017;10:1-5.

34. Hirva S, Jenisha P. Bicelle: a lipid nanostructure for transdermal delivery. J Crit Rev 2016;3:17-22.

\section{How to cite this article}

- Delly Ramadon, Seshiana Sebti Pramesti, Effionora Anwar. Formulation, stability test and in vitro penetration study of transethosomal gel containing green tea(Camellia sinensis L. kuntze) leaves extract. Int J Appl Pharm 2017;9(5):91-96. 